\title{
Batch Processing Method in Machine to Machine Wireless Communication as Smart and Intelligent System
}

\author{
Nurul Hiron, Asep Andang, and Hatib Setiawan
}

\begin{abstract}
This study discusses the performance of wireless communication between machines as smart and intelligent systems. The research involves the host-node as a receiver of capture temperature sensing and humidity sensors as sensor-nodes. The purpose of this study is the evaluation of the wireless communication performance as sensor-node sending data to the host in different distance between the sensor to the host-node by implementation batch processing method. In this research involves batch processing methods as management of digital data transmission in microprocessor ATMega. Using the ZigBeeIEEE 802.15.4 as communication protocol, and the temperature sensors and humidity sensor useing DHT2. In communications test, with the condition without any obstacles between sensor nodes to a host node. found that, at a distance of 18 meters, wireless data communications obtained by the successful delivery of data of $100 \%$. While at a distance of 58.18 meters to send data success rate decreased to $95 \%$ and at a distance of $107 \mathrm{~m}$ success rate of sending data down to only $69 \%$. This study illustrates that, batch processing method only guarantee the security of data sent, but does not affect the quality of wireless communication between machines.at a distance of 107 meters transmit data rate of success is $69 \%$.
\end{abstract}

Index Terms-Batch processing, wireless, intelligent system.

\section{INTRODUCTION}

Communication between machines that are used as smart and intelligent system depends on the quality of the communication that occurs between the sensor and its server. Smart and intelligent communications system, according to the medium is divided into two main types, it is a wire-based and wireless-based. Based on wireless communication, digital data management becomes the most important part. A wireless communication technology that is currently widely used, to some extent so that the technology is evolving rapidly one of which is the Internet of Things (IOT) where control and monitoring equipment to support human needs based on Internet technology.

Smart and intelligent system based on wireless communication, depending on how to treat the data capture at the sensor node, so that the communication between the sensor nodes and host nodes can take place according to the desire. Batch processing method has an important role in communication, in which the method changes data capture into multiple data packets and stored on the memory before it is sent to the host node or server.

There are some categories of data acquisition method,

Manuscript received March 5, 2016; revised June 14, 2016.

The authors are with the Department of Electrical Engineering, University of Siliwangi, Tasikmalaya, West Java, Indonesia (e-mail: hiron@unsil.ac.id). including continuous, batch, semi-batch [1] and sequential [2]. Continues process commonly used in systems analysis which involves the integration of the two concepts, namely Statistical Process Control (SPC) and engineering process control (EPC) [3]. Batch processing is used in multi-sensor communication system known as Wireless Sensor Network (WSN) to improve the efficiency of the performance of the WSN [4].

Equation batch processing, according to Soloviev [2] is as follows:

$$
\widehat{X}_{n}=\sum_{k=1}^{n} \alpha_{k} \cdot \tilde{X}_{k}
$$

where $\bar{x}_{\mathrm{k}}$ is a data measurement and $\alpha$ is a constant. The above equation states only occur if the process reaches the value of " $n$ " data. ZigBee protocol including the IEEE 802.15 family of standards along with Bluetooth (802.15.1) and WPAN (802.15.3) with the IEEE 802.15.4 standard code [5].

ZigBee network topology also has a "mesh" network so as to form a wider and more reliable data [6]. The advantages of ZigBee is operating at low power [7]. ZigBee standards applied to many embedded systems (embedded applications) such as industrial use [8], home automation systems [9]. Compared Bluetooth ZigBee has a lower transmission speed, which is $250 \mathrm{Kbps}$ with the communication distance up to 200 meters, whereas Bluetooth only reach 10 meters.

TABLE I: DifFERENCES BETWEEN UWB, BLUETOOTH, AND ZigBEE [10]

\begin{tabular}{lll}
\hline Standard & Bandwidth & Applications \\
\hline WPAN & $\begin{array}{l}\text { Up to } 54 \\
\text { Mbps }\end{array}$ & $\begin{array}{l}\text { Internet } \\
\text { browsing, } \\
\text { file transfers }\end{array}$ \\
Bluetooth & $1 \mathrm{Mbps}$ & $\begin{array}{l}\text { Wireless } \\
\text { USB, }\end{array}$ \\
ZigBee & $250 \mathrm{Kbps}$ & $\begin{array}{l}\text { Remote } \\
\text { control, } \\
\text { sensors }\end{array}$ \\
\hline
\end{tabular}

From the above discussion of some of the research, found that Zigbee with limitations deliver digital data. Digital data acquisition on the sensor node using batch processing method, will provide the successful delivery of data packets from the sensor node to node is hosted on a building wall hitch and distance variation.

\section{METHODOLOGY}

Measurement data transfer and signal strength between the sensor nodes and host nodes using X-CTU applications.

The data capture from the sensor-node is limited to 185 bytes of data sampling consists of 5 temperature and humidity 
5 sampling data, while sampling a complement data packets that shows the sampling time. The range of characters at each sampling is as much as 26 characters as Fig. 2 below, following Equation (1). Fig. 1 is schematic wireless communication sensor node and host node. Host node consists of a computer equipped with X-CTU program.

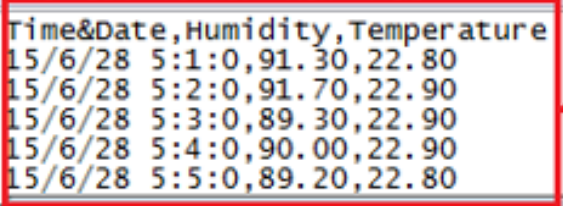

Fig. 1. Data packet sampling.

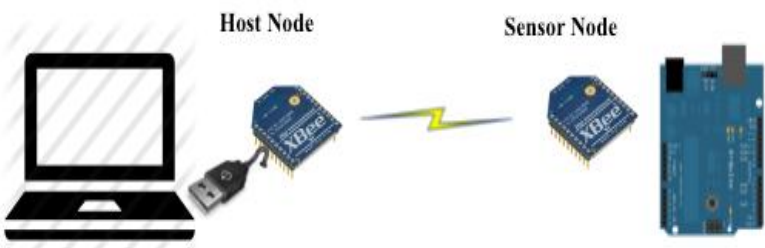

Fig. 2. Schematic wireless communication.

Data from read temperature and humidity through the stages of batch processing as Equation (1). Fig. 3 explain the stages of batch processing through the process on the sensor node schematic below:

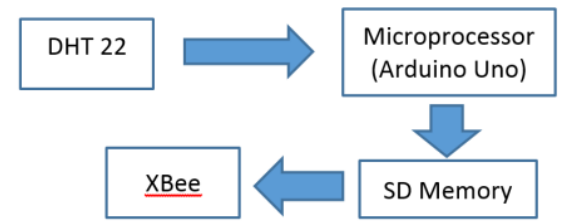

Fig. 3. Schematic batch process on the sensor node.

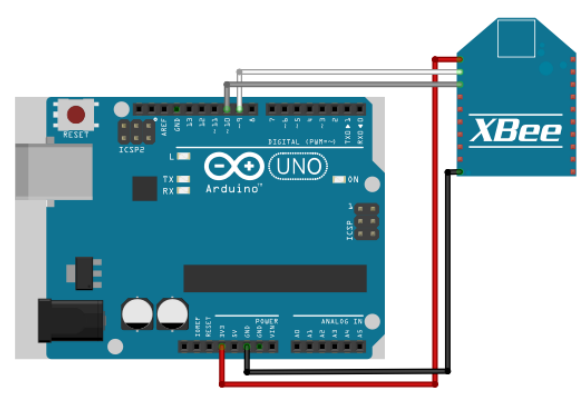

(a)
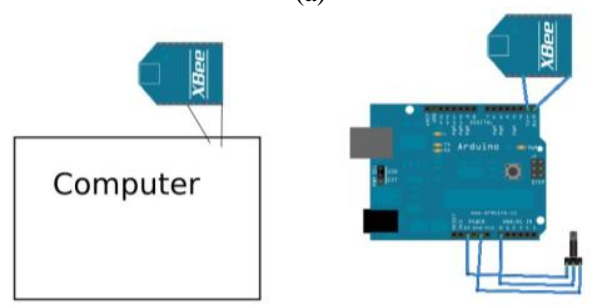

(b)

Fig. 4. (a) Wiring diagram XBee as sensor node. (b) Host node wiring schematic.

The process of data storage on a memory device (SD memory) through the command syntax is based on the equation (1) as follows:

if $(($ now.minute ()$\% 1==0 \& \&$ now.second ()$==0)$ || (now.minute ()$\% 1==0$ \&\& now.second ()$==1))\}$
Syntax above states that the sampling data stored in the SD card memory per each minute, while measuring data transmission command syntax as follows:

$\{$ if $(($ now.minute ()$\% 5==0 \& \&$ now.second ()$==0)$
$\|($ now.minute ()$\% 5==0 \& \&$ now.second ()$==1))\}$.

Syntax above states that measuring data sampling activities will be limited to once every 5 minutes, and then sent to the host node.

The schematically of sensor nodes design, as shown in Fig. 4 (a) is a wiring sensor nodes which consist of modules XBee, Arduino Uno as a receiver of data packets from the sensor node and Fig. 4 (b) shows a schematic wiring host node and Wiring Diagram XBee as Host Node.

\section{RESULT}

Results of this study resulted in several things including communication of test results at varying distances with singal strength measurements using X-CTU program as host nodes. Tests held at a distance of 1 meter to 110 meters, then measure the signal strength, the success of the data sent, the number of data are sent, and the number of errors that occur during transmission at several different distances.

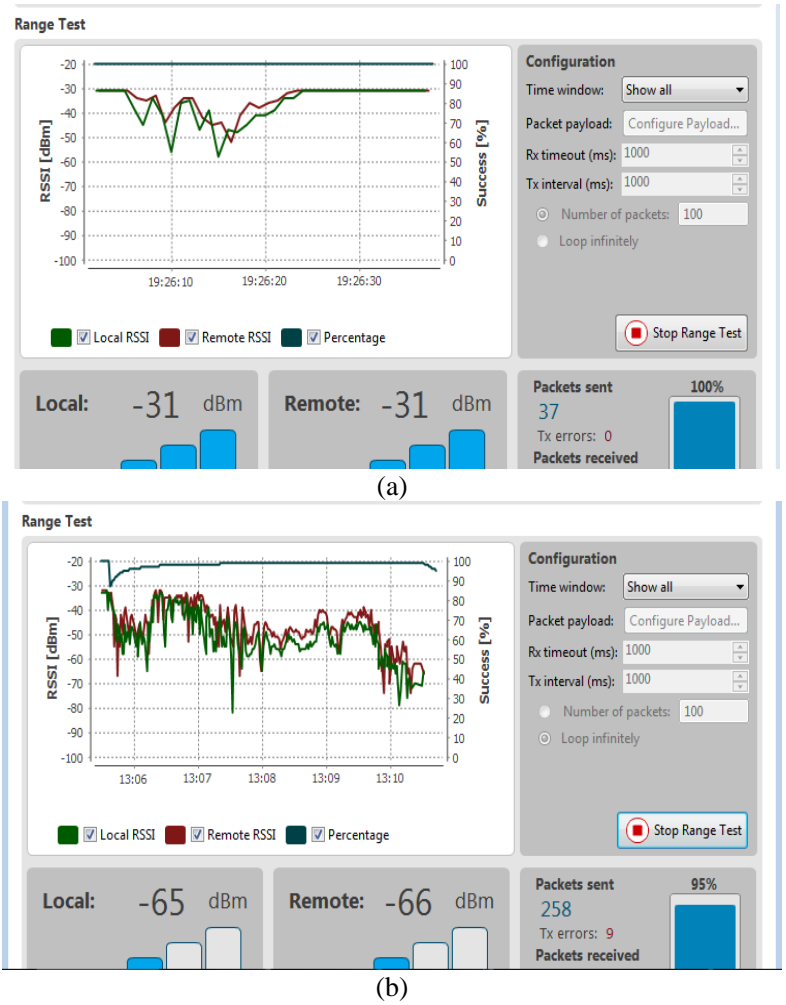

Fig. 5. Results of data transfers at a distance of 18.5 meters (a) and 58,18 meters (b).

Fig. 5(a) shows the analysis results of the sensor node communication to the host node at a distance of 18.5 meters with 37 packet data sent from sensor node, detected signal strength of $-31 \mathrm{dBm}$, percentage of packet delivery failure is $0 \%$. It is mean that the package of sensor nodes $100 \%$ accepted by the host node. Fig. 6 (b) shows the analysis results of the sensor node communication to the host node at a distance of 58.18 meters to 258 data packets. at this distance, 
there was $95 \%$ packet of data sent by a powerful signal smaller, which is $-65 \mathrm{dBm}$ to $-66 \mathrm{dBm}$ at the local and remote. Errors condition that occur during data communication is 9 times.
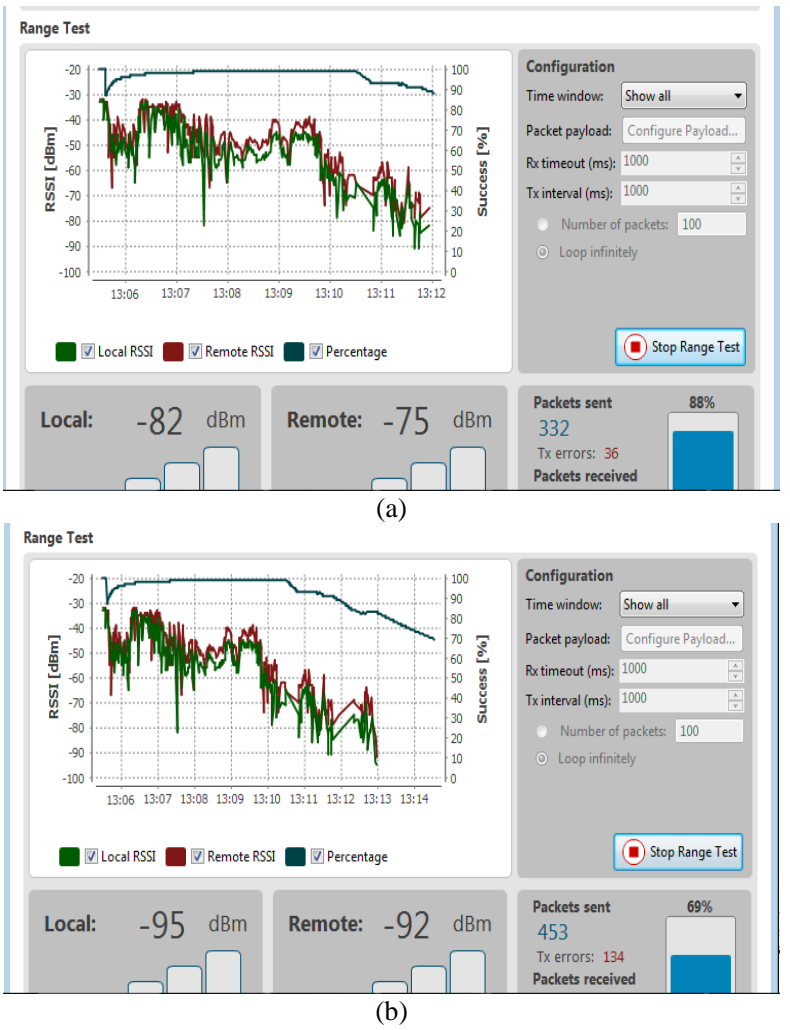

Fig. 6. Results of data transfers at a distance of 88,83 meters (a), 107,52 meters (b).

Fig. 6 (a) shows the analysis results of the sensor node communication to the host node at a distance of 88.83 meters to 332 data packets. at this distance, there was $88 \%$ packet of data sent by a powerful signal smaller, which is $-82 \mathrm{dBm}$ to $-75 \mathrm{dBm}$ at the local and remote. Errors that occur during data communication is 36 times. Fig. 6 (b) shows the analysis results of the sensor node communication to the host node at a distance of 107,52 meters to 453 data packets. at this distance, there was $69 \%$ packet of data sent by a powerful signal smaller, which is $-95 \mathrm{dBm}$ to $-92 \mathrm{dBm}$ at the local and remote. Errors that occur during data communication is 134 times.

TABLE II: SUMMARY OF TEST RESULTS

\begin{tabular}{|c|c|c|c|c|}
\hline \multirow{2}{*}{$\begin{array}{c}\text { Distance } \\
\text { (meter) }\end{array}$} & \multicolumn{2}{|c|}{ Signal (dBm) } & \multirow{2}{*}{$\begin{array}{c}\text { Data } \\
\text { packet sent } \\
(\%)\end{array}$} & \multirow[b]{2}{*}{ Error } \\
\hline & $\begin{array}{l}\text { Host } \\
\text { Node }\end{array}$ & $\begin{array}{c}\text { Sensor } \\
\text { Node }\end{array}$ & & \\
\hline $\pm 18,5$ & -31 & 100 & 100 & 0 \\
\hline $\pm 58,18$ & -65 & 95 & 95 & 9 \\
\hline $\pm 88,83$ & -82 & 88 & 88 & 36 \\
\hline $\pm 107,52$ & -95 & 69 & 69 & 134 \\
\hline
\end{tabular}

Table II Shows the overall results of test data transmission from the sensor nodes to a host node based on variations in the distance with units of meters.

From some of the above test shows the strength of the signal, the error rate and the amount of data needed to send the wireless communication between the sensor nodes to the host node is proportional to the distance. the greater the distance between sensor nodes to a host node will require more power, while the data packets to be increased due to address the failure of data delivery started. These conditions force sensor nodes to work harder and require more energy.

\section{CONCLUSION}

Smart and intelligent systems based wireless communication with Zigbee protocol in fact depends on how the anticipation of failure in communication. Batch processing method plays an important role in these conditions. batch processing methods, in addition to changing data capture into data packets, also applied for would allow the backup data stored on a memory card, so it can access them offline.

X-CTU program installed on the computer can be used to measure the communication performance sensor node and the host node with Zigbee protocol. Parameters that can be measured is the success of the data sent, the number of data packets, errors that occur during transmission, and signal strength for communication to occur.

Communications test, with the condition without any obstacles between sensor nodes to a host node. found that, at a distance of 18 meters, wireless data communications obtained by the successful delivery of data of $100 \%$. While at a distance of 58.18 meters to send data success rate decreased to $95 \%$ and at a distance of $107 \mathrm{~m}$ success rate of sending data down to only $69 \%$. This study illustrates that, batch processing method only guarantee the security of data sent, but does not affect the quality of wireless communication between machines.

\section{ACKNOWLEDGMENT}

Variations barriers need to be involved in future studies, where some kind of barrier to contain several different materials will affect the quality of communication is different Wall material, metal, type of fluid, and the wood will give effect to the signal sensor nodes to a host node.

\section{REFERENCES}

[1] C. L. Smith. Control of Batch Processes, John Wiley \& Sons, Inc. Hoboken, New Jersey, 2014, ch. 3, pp. 71-72.

[2] A. Soloviev, "Utilizing batch processing for GNSS signal tracking," Ohio University, Feb. 27, 2007, Avionics Engineering Center, Published in: Calgary, Canada.

[3] W. Hachicha and I. Moussa, "Integration of statistical and engineering process control in a batch processes monitoring: case of alkyd polymerization reactor," International Journal of Control and Automation, vol. 5, no. 1, 2012.

[4] J. Trevathan, H. Ghodosi, and T. Myers, "Efficient batch authentication for hierarchical wireless sensor networks," presented at Seventh International Conference on Intelligent Sensors, Sensor Networks and Information Processing (ISSNIP), November 2011.

[5] S. A. Desai, P. H. Zope, and S. R. Suralka, "A review on IEEE 802.15.4- Standard for wireless body network," International Journal of Innovative Research in Science, Engineering and Technology, May 2013.

[6] P. Kinney, "Zigbee technology: Wireless control that simply works," presented at Communications Design Conference, 2003.

[7] J. Wang, M. Chen, and V. C.M. Leung, "Forming priority based and energy balanced ZigBee networks-a pricing approach," Springer Science+Business Media, September 2011

[8] N. A. Somani and Y. Patel, "Zigbee: A low power wireless technology for industrial applications," International Journal of Control Theory and Computer Modelling (IJCTCM), vol. 2, no. 3, May 2012. 
[9] T. Obaid, H. Rashed, A. Abou-Elnour, M. Rehan, M. M. Saleh, and M. Tarique, "Zigbee technology and its application in wireless home automation systems: A survey," International Journal of Computer Networks \& Communications (IJCNC), vol. 6, no. 4, July 2014.

[10] S. S. R. Ahamed, "The role of ZigBee technology in future data communication system," Journal of Theoretical and Applied Information Technology, Sathak Institute of Technology, p. 134, 2009.

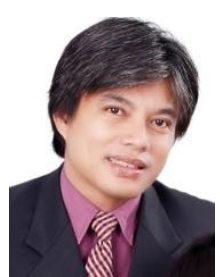

Nurul Hiron was born in Balikpapan, East Kalimantan, Indonesia, on August 19, 1975. He is a lecturer at the Electrical Engineering Department in Siliwangi University. His office is located in Tasikmalaya region in West Java province, Indonesia $\mathrm{He}$ started teaching in Electrical Engineering Departement from 2003 until now. He is a lecturer of energy management, microcontroller, electrical circuit, socio-technology and research methodology. His research interests include energy management, renewable energy, control, sensing, and human to machine interactions. He received his BS from Siliwangi (UNSIL) University Tasikmalaya and the MS from Gadja Mada University (UGM) Yogyakarta and the master of management from University Siliwangi (UNSIL) Tasikmalaya in Indonesia.

Asep Andang is a lecturer at the Electrical Engineering Department in Siliwangi University. Asep Andang's office is located in Tasikmalaya region in West Java province, Indonesia. Asep Andang started teaching in Electrical Engineering Departement from 2000 until now. Asep Andang is a lecturer of power electronics, electrical machines, transmission system analysis. Asep Andang's research interests include power electronics, controller, sensing. He received his BS from Siliwangi (UNSIL) University Tasikmalaya and the MS from Bandung Institute of Technology (ITB) Bandung in Indonesia.

Hatib Setiawan is an electrical engineering student Univeritas Siliwangi Hatib Setiawan's research interest is microcontroller. 